\title{
Anti-oxidant Effects of Kiwi Fruit in Vitro and in Vivo
}

\author{
Haruyo Iwasawa, ${ }^{* a}$ Erika Morita, ${ }^{b}$ Satoru Yui, ${ }^{b}$ and Masatoshi Yamazaki ${ }^{b}$ \\ ${ }^{a}$ Center for Educational Research, Faculty of Pharmaceutical Sciences, Teikyo University; 2-11-1 Kaga, Itabashi-ku, \\ Tokyo 173-8605, Japan: and ${ }^{b}$ Department of Medical Life Chemistry, Faculty of Pharmaceutical Sciences, Teikyo \\ University; 1091-1 Suwarashi, Midori-ku, Sagamihara, Kanagawa 252-5195, Japan. \\ Received August 5, 2010; accepted October 21, 2010
}

\begin{abstract}
We previously reported that kiwi fruit is rich in polyphenols and has immunostimulatory activity. Polyphenols are widely known for having anti-oxidant effects. We also revealed potential anti-oxidant effects of kiwi fruit in vivo by oral administration to mice. Here, we compared the anti-oxidant effects of kiwi fruit with those of other fruits in vitro. Then, we examined the inhibitory effects of kiwi fruit on oxidation in the human body. There are two varieties of kiwi fruit, green kiwi and gold kiwi. We also examined variation between these varieties. Comparison of the anti-oxidant effects in vitro demonstrated that kiwi fruit had stronger anti-oxidant effects than orange and grapefruit, which are rich in vitamin $C$; gold kiwi had the strongest anti-oxidant effects. Kiwi fruit inhibited oxidation of biological substances in the human body. In particular, kiwi fruit may inhibit early lipid oxidation. In this study, kiwi fruit had strong anti-oxidant effects and may prevent the development and deterioration of diseases caused by oxidative stress.
\end{abstract}

Key words kiwi fruit; anti-oxidant effect; 8-hydroxy-2'-deoxyguanosine; $N$ - $\varepsilon$-(hexanoyl)-lysine; polyphenol

Many studies have recently been conducted on functional food ingredients. Various in vivo benefits have been reported. ${ }^{1-4)}$ Many studies have been conducted on the antioxidant effects of polyphenol, one of the functional ingredients (i.e., phytochemicals). ${ }^{5-7)} \mathrm{We}$ also reported anti-oxidant, anti-inflammatory, anti-allergy, and anti-cancer effects of phytochemicals. ${ }^{8-12)}$ Various factors, such as inflammation, infection, stress, and drugs promote the production of active oxygen and free radicals in vivo. An appropriate amount of active oxygen seems to have beneficial effects, such as immunostimulation, within the living body. However, an excessive amount of active oxygen causes oxidative imbalance and attacks biological substances, resulting in oxidization of lipid, sugar, and protein, enzyme inactivation, DNA cleavage, and base oxidation. Oxides that are generated oxidize other biological substances and worsen the damage. This damages biomembranes and genes to decrease cellular functions, resulting in the development and deterioration of various diseases, such as arteriosclerosis, cancer, and diabetes. ${ }^{13)}$ Thus, inhibiting the generation of an excessive amount of active oxygen seems important to prevent the development and deterioration of various diseases.

We have already examined the correlation between various foods and immunity, particularly immunostimulatory action. ${ }^{14-16)}$ Kiwi fruit was one of the foods found to have immunostimulatory activity. Kiwi fruit is rich in vitamins and polyphenols and has strong anti-oxidant effects. We also reported that kiwi fruit is rich in polyphenols compared with other fruits. ${ }^{17)}$ We orally administered kiwi fruit to mice to examine its inhibitory effects on the generation of oxides in vivo. The results demonstrated that the oral administration of kiwi fruit reduced oxidative stress markers. ${ }^{16)}$ Anti-oxidant effects of kiwi fruit in vitro have been reported. ${ }^{18,19)}$ However, few reports have been published on studies comparing the anti-oxidant effects among fruits using several indicators.

Here, we compared the anti-oxidant effects among fruits popular in the marketplace, including kiwi fruit. In addition, we examined the anti-oxidant effects of kiwi fruit in vivo in healthy volunteers using the inhibitory effects on DNA and lipid oxidation as indicators. As an indicator of inhibitory effects on DNA oxidation we used 8-hydroxy-2'-deoxyguanosine $(8-\mathrm{OHdG})$. Inhibitory effects on lipid oxidation were examined using $N$ - $\varepsilon$-(hexanoyl)-lysine (HEL) as an indicator, which has recently attracted attention as a marker to detect early lipid peroxidation. According to a recent report, 8OHdG is decreased by the intake of foods containing antioxidants. ${ }^{20,21)}$ Several reports have been published on kiwi fruit in relation to allergy. ${ }^{22-24)}$ In addition, a report has been recently published on $8-\mathrm{OHdG},{ }^{25)}$ but not on HEL after the intake of kiwi fruit. Nowadays, metabolic syndrome is attracting a lot of attention. We considered that the anti-oxidant effects of kiwi fruit in the human body may prevent the development and deterioration of diseases caused by oxidative stress.

\section{MATERIALS AND METHODS}

Fruits For this study, we selected Hayward (Actinidia deliciosa), the most common commercially available breed of kiwi, Zespri green kiwi, and Hort16A (Actinidia chinensis), which is known as Zespri gold kiwi. These kiwi fruits (production area is New Zealand) were donated by Zespri Co., Ltd. The flesh was weighed, cut into appropriate sizes, and mixed using a mixer for $30 \mathrm{~s}$. Each homogenate was centrifuged (High-capacity refrigerated centrifuge 8800, Kubota Co., Ltd., Tokyo, Japan) at $3000 \mathrm{rpm}$ for $10 \mathrm{~min}$, and the resulting supernatant was passed through a filter. Each supernatant was centrifuged at $16000 \mathrm{rpm}$ for $1 \mathrm{~h}$ (Automatic highspeed refrigerated centrifuge 20PR-52, Hitachi Koki Co., Ltd., Tokyo, Japan), and the suspended substances were removed to obtain the sample solution. Fruits for examination of anti-oxidant effects in vitro were purchased at a food store. We selected five frequently consumed fruits besides kiwi fruit. These were apple (production area is Aomori, Japan), navel orange (U.S.A.), mandarin orange (Tokushima, Japan), white grapefruit (Florida, U.S.A.), and ruby grapefruit (Florida, U.S.A.). The flesh was weighed, cut into appropriate sizes, and mixed using a mixer for $30 \mathrm{~s}$. Each homogenate 
was centrifuged at $3000 \mathrm{rpm}$ for $10 \mathrm{~min}$, and the resulting supernatant was passed through a filter. Each supernatant was centrifuged at $16000 \mathrm{rpm}$ for $1 \mathrm{~h}$, and the suspended substances were removed to obtain the sample solution.

Detection of Polyphenols in Samples Polyphenols were detected by the Folin-Ciocalteu method, a routine detection method. ${ }^{26)}$ As a control sample, (-)-epicatechin (Wako Pure Chemical Industries, Osaka, Japan) was used.

Comparison of the Amounts of Oxidized Lipid Generated by UV Irradiation for Different Time Periods Each test sample $(100 \mu \mathrm{l})$ was added to $300 \mu \mathrm{l}$ of $2 \mathrm{mg} / \mathrm{ml}$ phosphatidyl ethanolamine (L- $\alpha$-phosphatidyl ethanolamine solution from egg yolk, Wako Pure Chemical Industries, Osaka, Japan) in test tubes. After mixing, the samples were UV-irradiated from above for 30, 60, 90, and $120 \mathrm{~min}$. Four test samples, green kiwi, gold kiwi, (-)-epicatechin, and deionized water (DIW) as a control, were prepared. Kiwi fruit was prepared at a final concentration of $5 \%$, and $(-)$-epicatechin at $1.5 \mathrm{mg} / \mathrm{ml}$, the same concentration as that on an average of green tea. ${ }^{27,28)}$ Oxidized lipid generated by UV irradiation was measured by the thiobarbituric acid-reactive substance (TBARS) test. ${ }^{29)}$

Inhibitory Effects on Lipid Oxidation in Vitro Phosphatidyl ethanolamine $(300 \mu \mathrm{l})$ and $100 \mu \mathrm{l}$ of each fruit sample were added to test tubes, followed by stirring and UV-irradiation for $90 \mathrm{~min}$. Fruit samples were prepared at a final concentration of $1 \%$. Deionized water, instead of a sample, was added as a control. Oxidized lipid generated by UV irradiation was measured by the TBARS test. The rate of inhibition of lipid oxidation was calculated using the following formula: inhibition rate of lipid oxidation $(\%)=(1-$ optical density (OD) sample/OD control) $\times 100$.

Elimination of $\mathbf{H}_{2} \mathbf{O}_{2}$ in Fruits Fruit samples were prepared at final concentrations of $1.25,5,10$, or $20 \%$ with DIW. As positive control, vitamin $\mathrm{C}$ and (-)-epicatechin were used. We set concentration of vitamin $C$ at $2.5 \mathrm{mg} / \mathrm{ml}$, corresponding to the content of green kiwi. ${ }^{30)}$ To these samples, $0.5 \mathrm{~mm} \mathrm{H}_{2} \mathrm{O}_{2}$ solution was added, followed by reaction at $37^{\circ} \mathrm{C}$ for $2 \mathrm{~h}$. This solution was reacted for $30 \mathrm{~min}$ at room temperature with an aqueous peroxide color reagent to which a ferrous ammonium solution was added. Then, $\mathrm{H}_{2} \mathrm{O}_{2}$ concentration was calculated by measurement of absorbance at $550 \mathrm{~nm}$. $\mathrm{H}_{2} \mathrm{O}_{2}$-eliminating activity was calculated using the following formula: $\mathrm{H}_{2} \mathrm{O}_{2}$-eliminating activity $(\%)=\left(1-\mathrm{H}_{2} \mathrm{O}_{2}\right.$ concentration of sample $/ \mathrm{H}_{2} \mathrm{O}_{2}$ concentration of control) $\times$ 100 .

Measurement of Superoxide Dismutase (SOD)-Like Activity in Fruits Fruit samples were prepared at a final concentration of $1 \%$ with DIW. SOD Assay Kit-WST (Dojindo Molecular Technologies, Inc., Kumamoto, Japan) was used to measure SOD activity (inhibition rate (\%)).

Measurement of Potential Anti-oxidant (PAO) in Fruits Potential anti-oxidant (PAO) kit (Japan Institute for the Control of Aging, Nikken Seil Co., Ltd., Tokyo, Japan) was used. This kit utilizes a reduction reaction of copper ions by antioxidants in a sample to determine total reducing power and anti-oxidant effects of the sample. The reducing power of uric acid is detected as a reference standard. Fruit samples were prepared at a final concentration of $1 \%$ with DIW.

Anti-oxidant Effects of Kiwi Fruit in Vivo in Healthy Volunteers Anti-oxidant effects of kiwi fruit were exam- ined in three healthy volunteers. Kiwi fruits were donated by Zespri Co., Ltd. The volunteers ate one kiwi fruit at a time, three times a day in the morning, noon, and evening for $7 \mathrm{~d}$. The volunteers started to eat kiwi fruit in the evening after urine sampling and ended the intake at noon on day 7. We designated "day 0" as the day when the volunteers started to eat kiwi fruit. Urine was collected at $2 \mathrm{~h}$ after the intake of kiwi fruit at noon. Urine was collected six times in total, before the start of intake (days -3 and 0 ), during intake (days 2, 4, and 7), and after intake (day 9). During this trial period (between day -3 and day 9), the volunteers ate neither other fruits nor supplements such as vitamins, and no vegetables were consumed in the morning or at noon on the days of urine collection. During the period, the same menu was used for lunch on the day of each urine collection. The volunteers drank only water as a beverage on the days of urine collection. During the period, the volunteers refrained from drinking beverages other than water wherever possible. Oxidative-stress markers excreted in urine (8-hydroxy-2' -deoxyguanosine: $8-\mathrm{OHdG}$ and $N$ - $\varepsilon$-(hexanoyl)-lysine: HEL) were measured as indicators of anti-oxidant effects of kiwi fruit. The amount of polyphenols in urine was also measured. Enzyme-linked immunosorbent assay (ELISA) kit (Japan Institute for the Control of Aging, Nikken Seil Co., Ltd., Fukuroi, Japan) was used to measure the oxidative stress markers. The Folin-Ciocalteu method was used to measure polyphenols in urine. To conduct this trial, we received approval from the Ethics Committee of Teikyo University, and explained the trial to the participants in writing and orally and obtained their consent.

\section{RESULTS}

Polyphenol Concentrations of Fruits The polyphenol concentrations of the fruit samples were compared. Gold kiwi contained the highest level of polyphenols $(1.04 \mathrm{mg} / \mathrm{ml})$, followed by green kiwi $(0.85 \mathrm{mg} / \mathrm{ml})$, navel orange, mandarin orange, grapefruit, and apple in this order (Fig. 1). To remove insoluble components, the samples were centrifuged at high speeds. Polyphenol reduction at different speeds (3000, $16000 \mathrm{rpm}$ ) was examined. After high-speed centrifugation, the same amounts of polyphenols were detected. Subsequently, we examined the changes in polyphenol concentration in heated kiwi fruit juice. Even after heating at $100^{\circ} \mathrm{C}$ for $60 \mathrm{~min}$, the polyphenol concentrations showed no change (date not shown). We also examined changes due to ripening. The polyphenol concentrations showed little change associated with hardness (date not shown). Thus, among the fruits, kiwi fruit was the richest in polyphenols. The polyphenols detected seemed relatively resistant to heat. After high-speed centrifugation, the extract became a transparent liquid with a faint color. This suggests that the components of kiwi fruit, other than pigment, may be involved in the effects identified in this study.

Inhibitory Effects of Kiwi Fruit on Lipid Oxidation To examine the proportion of oxidized lipid generated by UV irradiation, oxidized lipid was measured by the TBARS method for various time periods after irradiation. In the control with no anti-oxidant added, the oxidized lipid increased gradually up to $60 \mathrm{~min}$ and sharply at $90 \mathrm{~min}$. The production reached high level at $90 \mathrm{~min}$ and changed little at $120 \mathrm{~min}$ 


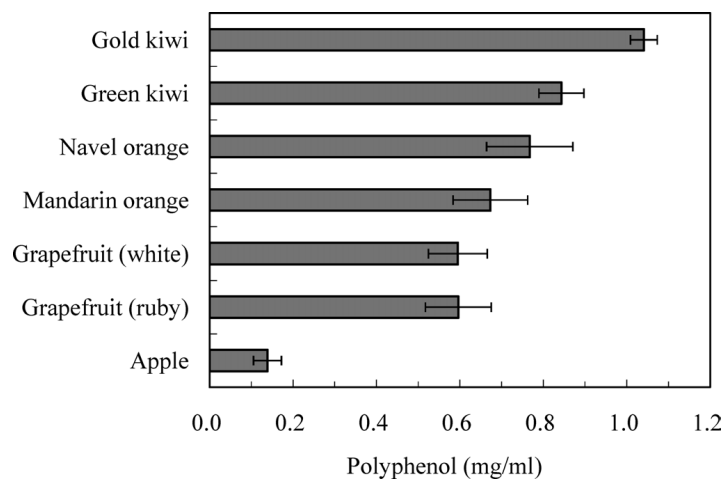

Fig. 1. Concentration of Polyphenols in Fruit Juices

The data are shown as means \pm S.D. $(n=6)$. Polyphenols were detected by the Folin-Ciocalteu method, a routine detection method. (-)-Epicatechin was used as a control sample. The $X$-axis represents the polyphenols concentration.

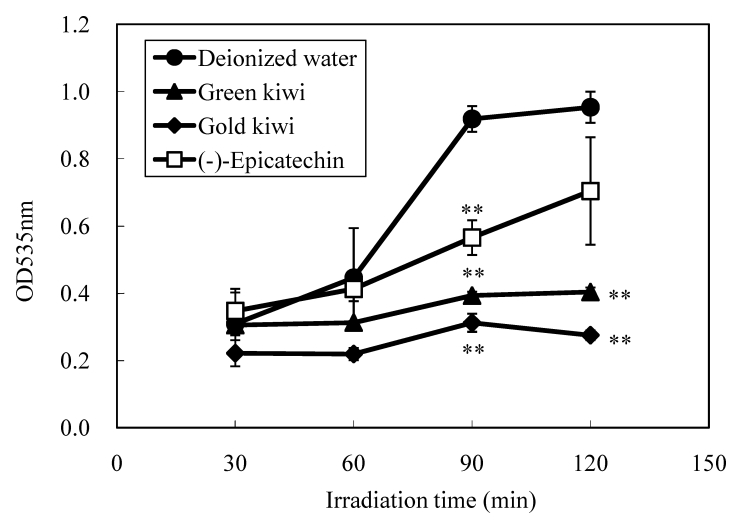

Fig. 2. Oxidized Lipid Generated by UV Irradiation over Time

The data are shown as means \pm S.D. $(n=4)$. Fruit samples were prepared at a final concentration of $5 \%$. The $X$-axis represents the period of UV irradiation. The $Y$-axi represents absorbance at $535 \mathrm{~nm}$. Statistical differences were analyzed by Dunnett's test. Significant differences were from comparisons with the control group. $* * p<0.01$.

(Fig. 2). After the addition of the same amount of (-)-epicatechin as that contained in tea, as another control, the production remained the same as that of the first control at $30 \mathrm{~min}$. Subsequently, the amount of oxidized lipid increased, but was as low as $74 \%$ of that of the first control even at $120 \mathrm{~min}$. In the case of green or gold kiwi fruit was added, the absorbance remained at the initial level even at $120 \mathrm{~min}$ (Fig. 2). The amount of oxidized lipid used in this study reached high level after UV irradiation for $90 \mathrm{~min}$. Thus, in the following experiments, UV irradiation was performed for $90 \mathrm{~min}$. The anti-oxidant effects of kiwi fruit showed no difference after heating at $100{ }^{\circ} \mathrm{C}$ for $60 \mathrm{~min}$ or between centrifugation speeds $(3000,16000 \mathrm{rpm})$ (data not shown). Kiwi fruit has an acidic $\mathrm{pH}(\mathrm{pH} 3)$. The effects of kiwi fruit may be influenced by the $\mathrm{pH}$ of the digestive tract. Thus, we examined the inhibitory effects on lipid oxidation at acidic to neutral $\mathrm{pH}$ levels. The results demonstrated that the effects were stronger at neutral $\mathrm{pH}$ than acidic $\mathrm{pH}$, although there was no significant difference (date not shown). These results were the same as those of polyphenol detection.

All the fruits showed dose-response patterns when their inhibitory effects on lipid oxidation were compared using their fruit juices. Green tea, known for its strong anti-oxidant effects, ${ }^{28)}$ also showed a dose-response pattern. All the $5 \%$ samples exhibited about an $80 \%$ inhibition of lipid oxidation;

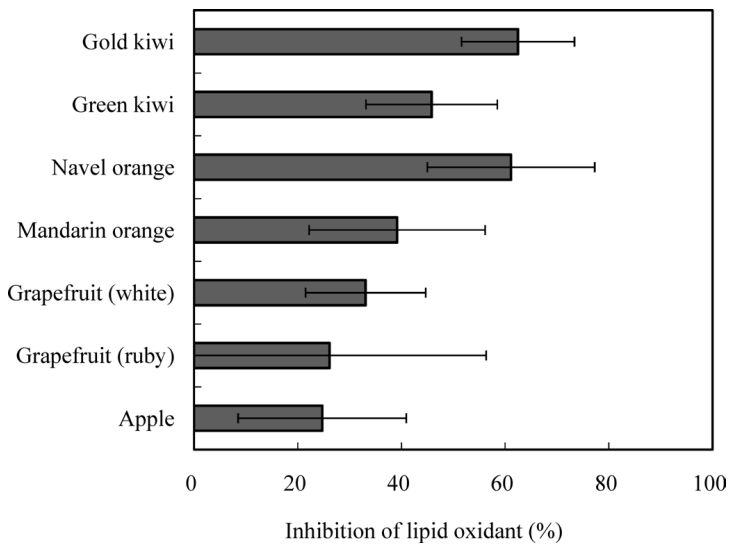

Fig. 3. Inhibition of Lipid Oxidant by Fruit Juices

The data are shown as means \pm S.D. $(n=16)$. The $X$-axis represents the proportion of inhibition of lipid oxidant. Fruit samples were prepared at a final concentration of $1 \%$. The rate of inhibition of lipid oxidation was calculated using the following formula: inhibition rate of lipid oxidation $(\%)=(1-$ OD sample/OD control $) \times 100$.

no significant difference was noted between different fruits (data not shown). However, $1 \%$ fruit juices had different rates of inhibition of lipid oxidation between different fruits. Gold kiwi and navel orange had the strongest inhibition rates of lipid oxidation, showing $60 \%$ even at $1 \%$ concentration. Green kiwi, mandarin orange, grapefruit, and apple followed in this order (Fig. 3). We show the results obtained using an L- $\alpha$-phosphatidyl ethanolamine solution from egg yolk. Similar results were obtained with phosphatidyl choline (L- $\alpha$ phosphatidyl choline in a chloroform solution from egg yolk, Sigma-Aldrich Co., Tokyo, Japan), which is abundant in the body (data not shown).

These results demonstrated that kiwi fruit, particularly gold kiwi, has strong inhibitory effects on lipid oxidation. The effects remained the same even after heating at $100{ }^{\circ} \mathrm{C}$ for $60 \mathrm{~min}$. Thus, active ingredients of kiwi fruit are resistant to heat. This seems to be associated with the fact that heating had no effect on the amount of polyphenol present. Thus, we considered that oxidation could be inhibited in the cell membrane of the intestine and during lipid absorption.

Comparison of $\mathrm{H}_{2} \mathrm{O}_{2}$-Eliminating Activities among Fruits Among indicators for use in comparing anti-oxidant effects, $\mathrm{H}_{2} \mathrm{O}_{2}$-eliminating activities of fruit juices were compared. Four concentrations between $1.25 \%$ and $20 \%$ were set for comparison. All the fruit juices, except for apple juice, had higher $\mathrm{H}_{2} \mathrm{O}_{2}$-eliminating activities as their concentration increased (date not shown). All the fruits of $20 \%$ concentration had $60 \%$ or above $\mathrm{H}_{2} \mathrm{O}_{2}$-eliminating activities. Apple juice of $20 \%$ concentration had no $\mathrm{H}_{2} \mathrm{O}_{2}$-eliminating activity. Gold kiwi and grapefruit had nearly $100 \%$ and green kiwi $80 \% \mathrm{H}_{2} \mathrm{O}_{2}$-eliminating activities. Unlike other fruits having $10-30 \% \quad \mathrm{H}_{2} \mathrm{O}_{2}$-eliminating activities, $5 \%$ gold kiwi juice had $70 \% \quad \mathrm{H}_{2} \mathrm{O}_{2}$-eliminating activity (Fig. 4). The same amount of vitamin $\mathrm{C}$ as that contained in green kiwi fruit and (-)-epicatechin contained in green tea were examined, and neither had $\mathrm{H}_{2} \mathrm{O}_{2}$-eliminating activity. These results demonstrated that gold kiwi had the highest $\mathrm{H}_{2} \mathrm{O}_{2}$-eliminating activity and that this activity resulted from ingredients such as polyphenols other than vitamin $\mathrm{C}$.

Comparison of SOD-Like Activities among Fruits We also examined superoxide dismutase (SOD)-like activity that 


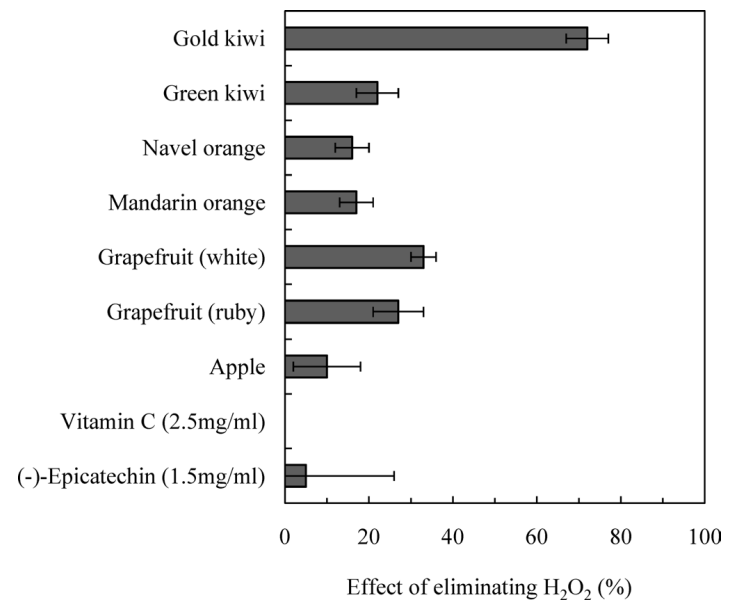

Fig. 4. Effects of Eliminating $\mathrm{H}_{2} \mathrm{O}_{2}$ by Fruit Juices

The data are shown as means \pm S.D. $(n=6)$. Fruit samples were prepared at a final concentration of $5 \%$. The $X$-axis represents proportion of eliminated $\mathrm{H}_{2} \mathrm{O}_{2} \cdot \mathrm{H}_{2} \mathrm{O}_{2}$-eliminating activity was calculated using the following formula: $\mathrm{H}_{2} \mathrm{O}_{2}$-eliminating activity $(\%)=\left(1-\mathrm{H}_{2} \mathrm{O}_{2}\right.$ concentration of sample $/ \mathrm{H}_{2} \mathrm{O}_{2}$ concentration of control $) \times 100$.

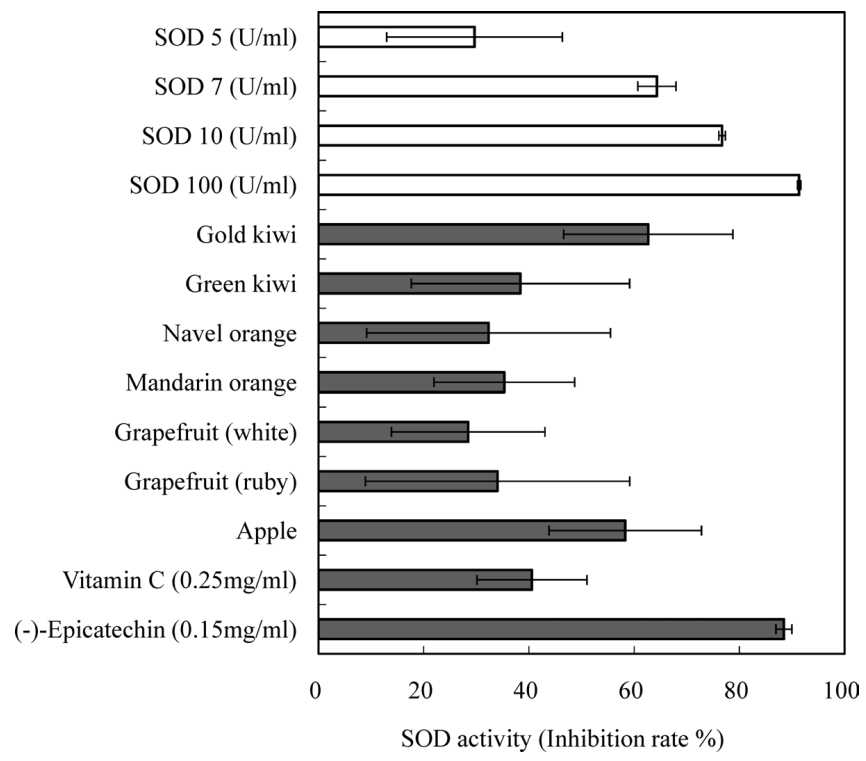

Fig. 5. SOD-Like Activity of Fruit Juices

The data are shown as means \pm S.D. $(n=6)$. The $X$-axis represents SOD activity (inhibition rate). Fruit samples were prepared at a final concentration of $1 \%$.

eliminates superoxide, a kind of active oxygen. The levels of activities were found to be in the following order: gold kiwi, apple, green kiwi, mandarin orange, grapefruit (ruby), navel orange, and grapefruit (white) (Fig. 5). Vitamin $\mathrm{C}$ also had this activity. (-)-Epicatechin had a markedly high activity. The SOD-like activities of the fruits also seemed attributable to vitamin $\mathrm{C}$. However, the activities were inconsistent with the ranked data of vitamin $\mathrm{C}$ concentrations (see Standard Tables of Food Composition in Japan, Fifth Revised and Enlarged Edition). This indicates that ingredients other than vitamin $\mathrm{C}$ also seemed attributable to the activity. Thus, polyphenols in the fruits seem to be main candidate for SODlike activities. Apple had strong SOD-like activity and other anti-oxidant effects were week. Although the details were not clear, we considered that apple included a type of polyphenols which have a strong SOD-like activity but weak other anti-oxidant effects.

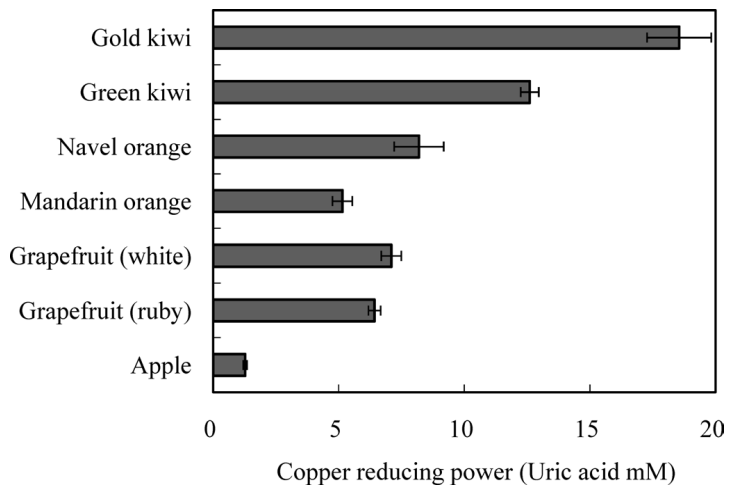

Fig. 6. Potential Anti-oxidant (PAO) Effects of Fruit Juices

The data are shown as means \pm S.D. $(n=4)$. The $X$-axis represents copper reducing power (uric acid $\mathrm{mm}$ ). Fruit sample were prepared at a final concentration of $1 \%$.

Comparison of Potential Anti-oxidants (PAO) among Fruits The copper-reducing powers of the fruits were reduced to those of uric acid for comparison. The results demonstrated that gold kiwi had the strongest reducing power, beyond twice the values obtained for the other fruits (Fig. 6). Green kiwi, navel orange, grapefruit, mandarin orange, and apple followed in this order. These results were inconsistent with the vitamin $\mathrm{C}$ concentrations. ${ }^{30)}$ Thus, polyphenols in the fruits seem to be main candidate for PAO activities.

Anti-oxidant Effects in Vivo in Healthy Volunteers An investigation on the anti-oxidant effects in vitro demonstrated that kiwi fruit had overall stronger anti-oxidant effects than the other fruits. A previous study by oral administration in mice suggested that kiwi fruit may have anti-oxidant effects in vivo. ${ }^{16}$ Thus, anti-oxidant effects were examined in healthy volunteers. Anti-oxidant effects were measured using oxidative stress markers excreted in urine, 8-hydroxy- $2^{\prime}$-deoxyguanosine (8-OHdG) and $N$ - $\varepsilon$-(hexanoyl)-lysine (HEL). $8-\mathrm{OHdG}$ is a relatively stable substance excreted in urine, which is cleaved on the oxidization of deoxyguanosine, a DNA base, and is used as an indicator of oxidative damage of DNA. Lipid peroxide generated from lipid oxidation binds to biomolecules, such as protein and nucleic acid. HEL is lipid peroxide bound to lysine in protein, a relatively stable product excreted in urine. HEL has recently attracted attention as an oxidative stress biomarker to detect early lipid peroxidation.

We performed a preliminary test on three volunteers prior to any trial on a larger number of volunteers. Volunteers were man in his late fifties and two women in her early thirties and early twenties. This test demonstrated a significant difference for gold kiwi. 8-OHdG was decreased from day 0 to day 4 and increased on day 7 or later (Fig. 7A). Significant differences were noted for volunteer "a" on days 4 and 7 and for volunteer "b" on days 2, 4, and 9. Volunteer "c" exhibited no significant differences. Over all, the decrease effects of 8-OHdG was not shown in green kiwi (Fig. 7B). HEL was decreased rapidly on day 2 and increased again from day 4 to day 7 , and on day 9 , and then returned to the same level as on day 0 on completion of intake of gold kiwi (Fig. 8A). Significant differences were noted for volunteer "a" on day 4 , for volunteer "b" on days 2, 4, and 7, and for volunteer "c" on day 2. Green kiwi showed significant reducing effects in vol- 
A

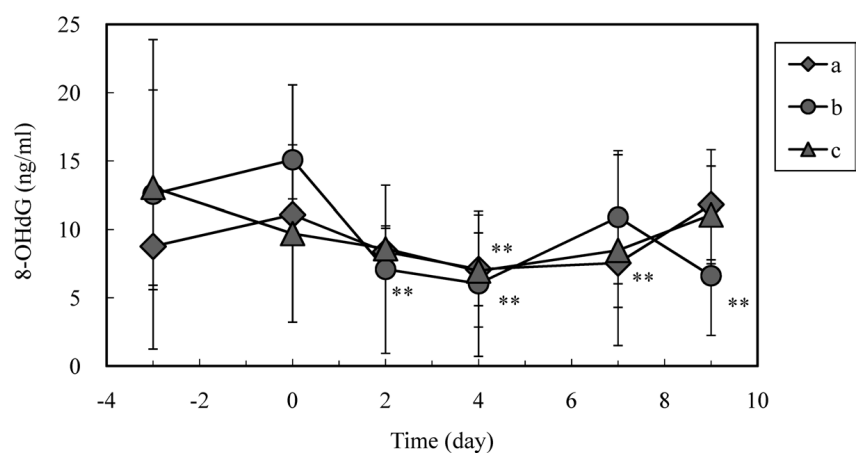

B

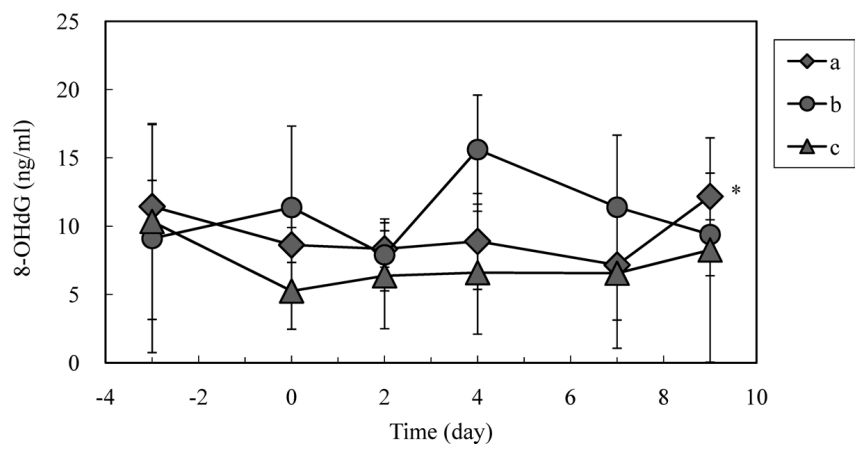

Fig. 7. Variation in Urinary 8-Hydroxy-2'-deoxyguanosine (8-OHdG) Levels after the Intake of Kiwi Fruit

The data are shown as means \pm S.D. $(n=3)$. "a," "b," and "c" represent data for the different volunteers. The $X$-axis represents the trial time. "Day -3 " and "day 0 " are before the intake of kiwi fruit. "Day 2" and "day 7" are days 2 and 7 after the intake of kiwi fruit, respectively. Kiwi fruits were consumed from the evening of day 0 to the afternoon of day 7. "Day 9" is after the intake of kiwi fruit. Statistical differences were analyzed by Dunnett's test. Significant differences were for each date compared with day $0 . * p<0.05, * * p<0.01$. A: Gold kiwi. B: Green kiwi.

unteer "b" (Fig. 8B). The effects of green kiwi and gold kiwi were compared using average data from the three volunteers. $8-\mathrm{OHdG}$ was decreased for green kiwi on day 2, although no significant difference was noted. $8-\mathrm{OHdG}$ was significantly decreased for gold kiwi on days 2, 4, and 7 (Fig. 9). HEL was significantly decreased for both green kiwi and gold kiwi on day 2, and the decreased levels were maintained up to day 7 (Fig. 10). On day 9, two days after completion of intake, HEL returned to the value it held on day 0 . We then examined whether polyphenols were excreted in urine because of the intake of kiwi fruit rich in polyphenols. The results demonstrated that the amount of polyphenols showed no change up to day 4, but increased on day 7 (Fig. 11). Successive intake for 1 week might be possible to increase polyphenols in urine on day 7 .

Thus, the intake of kiwi fruit had anti-oxidant effects in vivo. 8-OHdG was most effectively decreased on day 4 and HEL on day 2, although the timing of the exhibition of an effect varied among individuals. This seems to be associated with the fact that HEL is an early lipid peroxidation marker. Fruits may have inhibitory effects on lipid oxidation in the early stage after intake. Gold kiwi tended to have stronger effects than green kiwi. This is also consistent with the result that gold kiwi had stronger effects in vitro. Because suppressing early progression to arteriosclerosis may prevent the
A

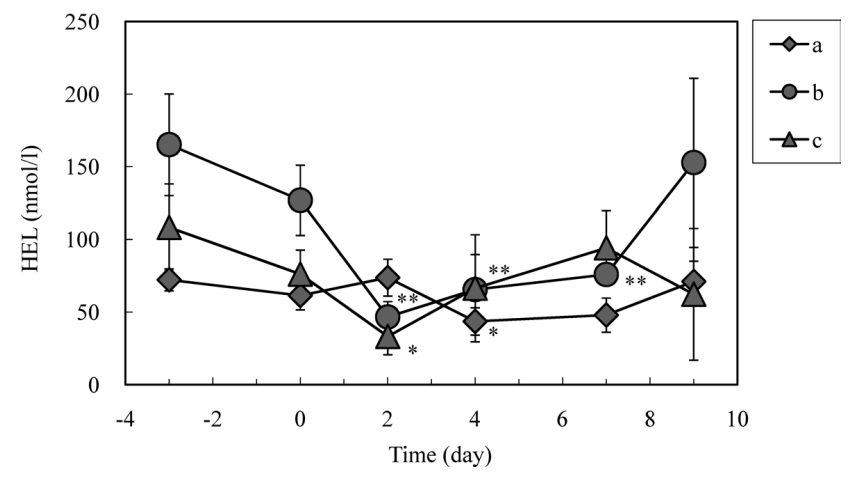

B

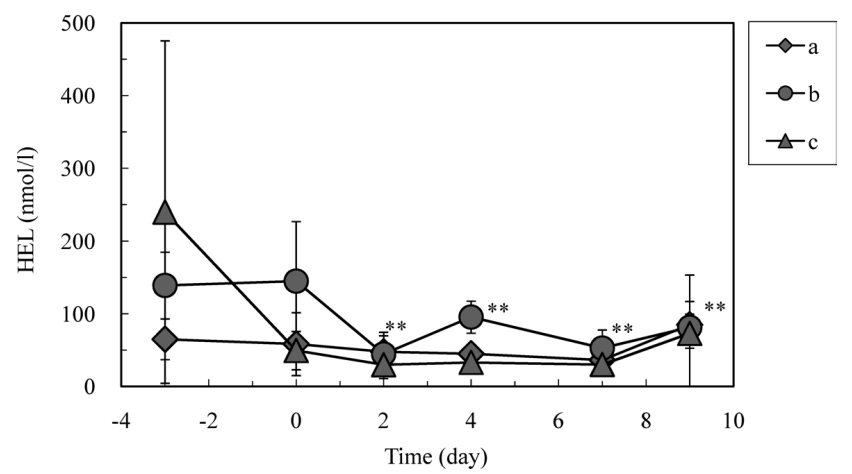

Fig. 8. Variation in Urinary $N$ - $\varepsilon$-(Hexanoyl)-lysine (HEL) Levels after the Intake of Kiwi Fruit

The data are shown as means \pm S.D. $(n=3)$. "a," "b," and "c" represent data for the different volunteers. The $X$-axis represents the trial time. "Day -3 " and "day 0" are before the intake of kiwi fruit "Day 2" and "day 7" are days 2 and 7 after the intake of kiwi fruit, respectively. Kiwi fruits were consumed from the evening of day 0 to the afternoon of day 7. "Day 9" is after the intake of kiwi fruit. Statistical differences were analyzed by Dunnett's test. Significant differences were for each date compared with day $0 . * p<0.05$, $* * p<0.01$. A: Gold kiwi. B: Green kiwi.

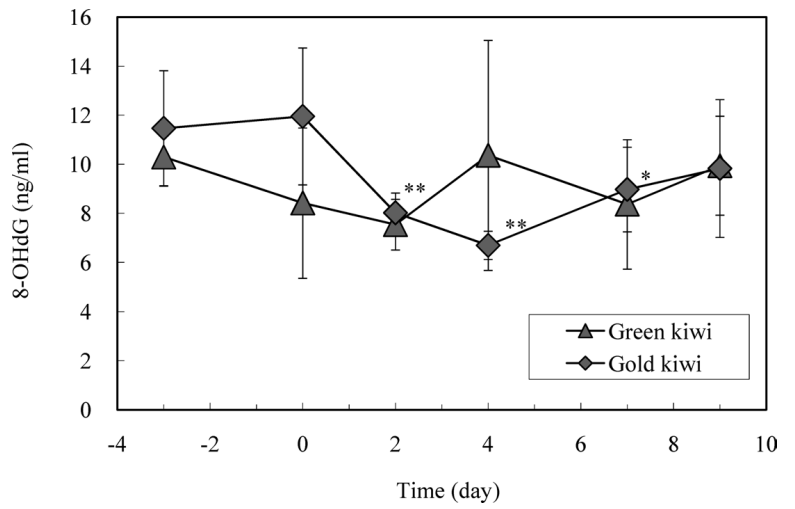

Fig. 9. Variation in Urinary 8-Hydroxy-2'-deoxyguanosine (8-OHdG) Levels after the Intake of Kiwi Fruits

The data are shown as means \pm S.D. $(n=9)$. The $X$-axis represents the trial time. "Day -3 " and "day 0" are before the intake of kiwi fruit. "Day 2" and "day 7" are days 2 and 7 after the intake of kiwi fruit, respectively. Kiwi fruits were consumed from the evening on day 0 to the afternoon on day 7. "Day 9" is after the intake of kiwi fruit These data are the averages for the three volunteers. Statistical differences were analyzed by Dunnett's test. Significant differences were for each date compared with day 0 . $* p<0.05, * * p<0.01$.

disease, the effects of eating gold kiwi may be particularly beneficial. 


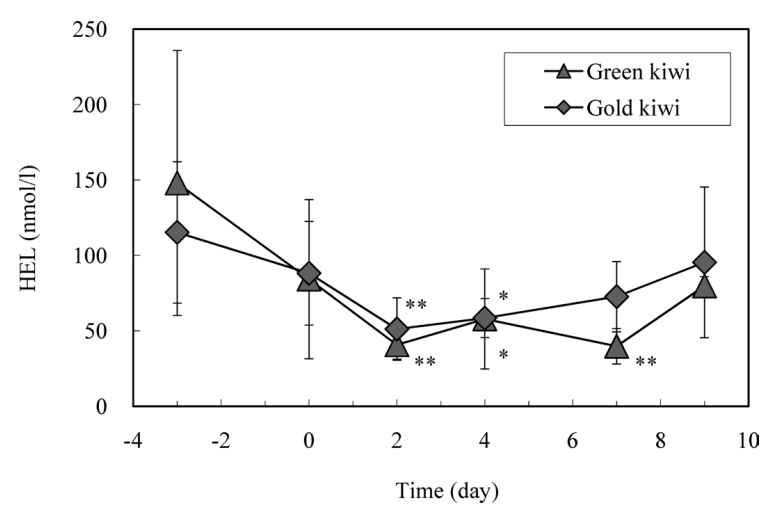

Fig. 10. Variation in Urinary $N$ - $\varepsilon$-(Hexanoyl)-lysine (HEL) Levels after per os (p.o.) Administration of Kiwi Fruit

The data are shown as means \pm S.D. $(n=9)$. The $X$-axis represents the trial time. "Day -3 " and "day 0" are before the intake of kiwi fruit. "Day 2" and "day 7" are days 2 and 7 after the intake of kiwi fruit, respectively. Kiwi fruits were consumed from the evening of day 0 to the afternoon of day 7. "Day 9" is after the intake of kiwi fruit. These data are the averages for the three volunteers. Statistical differences were analyzed by Dunnett's test. Significant differences were for each date compared with day 0 . $* p<0.05, * * p<0.01$.

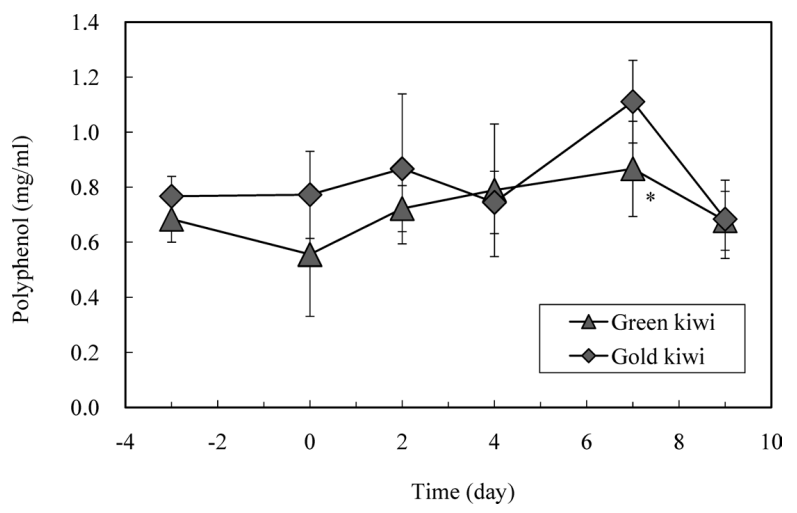

Fig. 11. Variation in Urinary Polyphenol Levels after p.o. Administration of Kiwi Fruit

The data are shown as means \pm S.D. $(n=9)$. The $X$-axis represents the trial time. "Day -3 " and "day 0" are before the intake of kiwi fruit. "Day 2" and "day 7" are days 2 and 7 after the intake of kiwi fruit, respectively. Kiwi fruits were consumed from the evening on day 0 to the afternoon on day 7. "Day 9" is after the intake of kiwi fruit. These data are the averages for the three volunteers. Statistical differences were analyzed by Dunnett's test. Significant differences were for each date compared with day 0 . $* p<0.05$.

\section{DISCUSSION}

Anti-oxidant effects in vitro were examined using four indicators, rate of inhibition of lipid oxidation, $\mathrm{H}_{2} \mathrm{O}_{2}$-eliminating effect, SOD-like activity, and PAO. All these indicators showed the highest activity for gold kiwi, demonstrating that gold kiwi has strong anti-oxidant effects (Table 1). Overall, green kiwi had lower anti-oxidant effects than gold kiwi, but had stronger effects than the other fruits. Some of the data concerning the ranking of anti-oxidant effects are inconsistent with those of polyphenol concentrations; however, they generally seem to be strongly associated. Reportedly, 8 -OHdG is increased with vitamin-deficient foods, suggesting that vitamins are an important ingredient for anti-oxidant effects. ${ }^{31)}$ Some indicators showed anti-oxidant effects of vitamin $\mathrm{C}$ in vitro, while others did not. Therefore, we considered that the strong anti-oxidant effects of kiwi fruit detected in this study were associated with the effects of polyphenols besides vitamins.
Table 1. Comparison of Anti-oxidant Activities among Various Fruits

\begin{tabular}{|c|c|}
\hline TBARS method & $\begin{array}{l}\text { Gold kiwi }>\text { navel orange }>\text { green kiwi }>\text { mandarin } \\
\text { orange }>\text { grapefruits }>\text { apple }\end{array}$ \\
\hline $\begin{array}{l}\mathrm{H}_{2} \mathrm{O}_{2} \text {-eliminating } \\
\text { activity }\end{array}$ & $\begin{array}{l}\text { Gold kiwi }>\text { grapefruits }>\text { green kiwi }>\text { mandarin } \\
\text { orange }>\text { navel orange }>\text { apple }\end{array}$ \\
\hline SOD-like activity & $\begin{array}{l}\text { Gold kiwi }>\text { apple }>\text { green kiwi }>\text { mandarin orange, } \\
\text { navel orange, grapefruits }\end{array}$ \\
\hline PAO & $\begin{array}{l}\text { Gold kiwi }>\text { green kiwi }>\text { navel orange, grapefruits }> \\
\text { mandarin orange }>\text { apple }\end{array}$ \\
\hline
\end{tabular}

We examined anti-oxidant effects using four indicators, TBARS method, $\mathrm{H}_{2} \mathrm{O}_{2}$-eliminating activity, SOD-like activity, and PAO. We ranked these effects among the fruits.

Here, we examined the anti-oxidant effects of kiwi fruit in vivo in healthy volunteers using the inhibitory effects on DNA and lipid oxidation as indicators. As an indicator of inhibitory effects on DNA oxidation we used 8-OHdG. 8OHdG is known to be increased in the urine of patients with diabetes or atherosclerosis, smokers, and cancer patients. ${ }^{32,33)}$ The inhibitory effects on lipid oxidation were examined using HEL as an indicator, which has recently attracted attention as a marker to detect early lipid peroxidation. A correlation between HEL and rheumatoid arthritis or arteriosclerosis has been reported. ${ }^{34,35)}$ In this study, 8-OHdG was most effectively decreased on day 4 and HEL on day 2, although the timing of the exhibition of these effects varied among individuals. Intake of three kiwi fruits per day in human is nearly equivalent applied dose of $1 \%$ kiwi fruit juices in mice. In consequence, we thought this trial design is appropriate. Both green kiwi and gold kiwi were different in many ways, for example sweetness and color of pulp. ${ }^{36)}$ However, both juices for in vitro experiments were a transparent liquid with a faint color, so we considered the components of kiwi fruit, other than pigment, may be involved in the anti-oxidant effects identified in this trial. Thus, the intake of kiwi fruit had anti-oxidant effects in vivo.

Arteriosclerosis is a disease that causes localized thickening, induration, and reconstruction of the arterial wall resulting in a reduction in function. Arteriosclerosis causes blood flow obstruction along with the progression of a lesion leading to diseases such as myocardial infarction. Oxidized lipid accumulates in an arteriosclerotic lesion. The degree of induration of the arteriosclerotic lesion is strongly correlated with the concentration of oxidized lipid. Denatured oxidized lipid is ingested by macrophages as a foreign substance. After a series of these reactions, macrophages turn into foam cells and proliferate to cause arteriosclerosis. ${ }^{37)}$ The ingesting capacity of macrophages and inhibitory effects on the production of oxidized lipid therefore seem important for the prevention of arteriosclerosis. We previously demonstrated that macrophages are activated by kiwi fruit. In addition, lipid oxidization in the presence of kiwi fruit can prevent macrophages from turning into foam cells. ${ }^{17)}$ Furthermore, we here demonstrated that the intake of kiwi fruit reduced oxides, particularly oxidized lipid, in vivo. Thus, the intake of kiwi fruit should inhibit oxidation of substances in the body and may prevent the development and deterioration of arteriosclerosis, particularly through inhibition of lipid oxidation and rapid ingestion by activated macrophages.

In addition, the intake of food with strong anti-oxidant effects is recommended because a meal containing only car- 
bohydrate, lipid, and protein was found to reduce the anti-oxidant effects after the meal. ${ }^{38)}$

Green kiwi (Hayward) extracts were reported to contain a number of anti-oxidant constituent such as vitamin $\mathrm{C}$ and $\mathrm{E}$, caffeic acid, naringenin, quercetin, epicathechin. ${ }^{39)}$ Some constituents were reported to have the inhibitory activity of xanthine oxidase or inducible nitric oxide synthase (iNOS). ${ }^{5}$ These anti-oxidant constituents may contribute to the strong anti-oxidant effects of kiwi fruits, suggesting the additive and synergistic effects of these factors. Anti-oxidant effects in disease such as hyperlipidemic subjects were reported, but few studies were reported in healthy volunteers. ${ }^{40,41)}$ And there is no report about these oxidative stress markers (8$\mathrm{OHdG}$ and HEL) in healthy volunteers. Although improvement of disease and/or prevention of disease progression may be important, prevention of incidence is also important. In this study, we clarified the decrease of oxidative stress markers in healthy volunteers by kiwi fruit intake. Therefore, we propose the novel possibility that daily consumption of kiwi fruit is effective on decrease of oxidative stress and further prevention of disease by excessive oxidation. We here reported a trial on a small number of subjects. In this trial, positive effects were observed, and no specific side effects were noted. Thus, we will conduct a trial on a larger number of subjects. The active ingredients are still unknown and need to be clarified.

\section{REFERENCES}

1) Fahey J. W., Zhang Y., Talalay P., Proc. Natl. Acad. Sci. U.S.A., 94, $10367-10372$ (1997).

2) Yu S., Paetan-Robinson I., Vet. Res. Commun., 30, 403 - 413 (2006).

3) Lee J. E., Giovannucci E., Smith-Warner S. A., Spiegelman D., Willett W. C., Curhan G. C., Cancer Epidemiol. Biomarkers Prev., 15, 2445 2452 (2006).

4) Shrestha S., Ehlers S. J., Lee J. Y., Fernandez M. L., Koo S. I., J. Nutr., 139, 640-645 (2009).

5) Tanaka T., Suzuki R., FFI J., 209, 472-481 (2004).

6) Yoshino K., Ihara H., Kimura S., Sugiura Y., J. Technol. Educ., 13, $1-$ 7 (2006).

7) Scalzo J., Politi A., Pellegrini N., Mezzetti B., Battino M., Nutrition, 21, 207-213 (2005).

8) Yamazaki M., Ueda H., "Perilla: the Genus Perilla," ed. by Yu H.-C., Kosuna K., Haga M., Harwood Academic Publishers, Amsterdam, 1997, pp. 47-54.

9) Ueda H., Yamazaki M., Biosci. Biotech. Biochem., 61, 1292-1295 (1997).

10) Yamazaki M., Ueda H., J. Nutr., 58, 101-108 (2000).

11) Ueda H., Yamazaki C., Yamazaki M., Biol. Pharm. Bull., 26, 560563 (2003).

12) Ueda H., Yamazaki C., Yamazaki M., Biol. Pharm. Bull., 25, $1197-$ 1202 (2002).
13) Kunitomo M., Yakugaku Zasshi, 127, 1997-2014 (2007).

14) Yamazaki M., Ueda H., "Food Factors for Cancer Prevention," ed. by Ohigashi H., Osawa T., Watanabe S., Yoshikawa T., Springer, New York, 1997, pp. 159-161.

15) Iwasawa H., Yamazaki M., Food Sci. Technol. Res., 15, 275-282 (2009).

16) Iwasawa H., Morita E., Ueda H., Yamazaki M., Food Sci. Technol. Res., 16, 135-142 (2010).

17) Yamazaki M., Ueda H., Yui S., Bio. Industry, 17, 24-31 (2000).

18) Collins B. H., Horská A., Hotten P. M., Riddoch C., Collins A. R., Nutr. Cancer, 39, 148-153 (2001).

19) Jung K. A., Song T. C., Han D., Kim I. H., Kim Y. E., Lee C. H., Biol. Pharm. Bull., 28, 1782-1785 (2005).

20) Thompson H. J., Heimendinger J., Haegele A., Sedlacek S. M., Gillette C., O’Neill C., Wolf P., Conry C., Carcinogenesis, 20, 2261-2266 (1999).

21) Dhawan V., Jain S., Mol. Cell Biochem., 275, 85-94 (2005).

22) Lucas J. S., Lewis S. A., Trewin J. B., Grimshaw K. E., Warner J. O., Hourihane J. O., Pediatr. Allergy Immunol., 16, 647-654 (2005).

23) Chen L., Lucas J. S., Hourihane J. O., Lindemamm J., Taylor S. L., Goodman R. E., Food Chem. Toxicol., 44, 1100-1107 (2006).

24) Bublin M., Mari A., Ebner C., Knulst A., Scheiner O., HoffmannSommergruber K., Breiteneder H., Radauer C., J. Allergy Clin. Immunol., 114, 1169-1175 (2004).

25) Collins A. R., Harrington V., Drea J., Melvin R., Carcinogenesis, 24, $511-515$ (2003).

26) Hatano T., "Development of Polyphenol-Rich Functional Foods," ed. by Yoshida T., Arii M., CMC Publishing, Tokyo, 2007, pp. 12-24.

27) Seto R., "Materials for Functional Food II," ed. by Ota M., CMC Publishing, Tokyo, 2001, pp. 225-228.

28) Cabrera C., Artacho R., Giménez R., J. Am. Coll. Nutr., 25, 79-99 (2006).

29) Kikukawa K., "Experimental Technique of Basic Biochemistry," Vol. 5, Chap. 7, ed. by the Japanese Biochemical Society, Tokyo Kagaku Dojin, Tokyo, pp. 54-57.

30) Kagawa Y., "Standard Tables of Food Composition in Japan, Fifth Revised and Enlarged Edition," Kagawa Nutrition University Publishing Division, Tokyo, 2008, pp. $94-95$.

31) Li Y. S., Kawai K., Kasai H., Gene. Environ., 29, 128-132 (2007).

32) Wu L. L., Chiou C. C., Chang P. Y., Wu J. T., Clin. Chim. Acta, 339, $1-9$ (2004).

33) Pilger A., Rudiger H. W., Int. Arch. Occup. Environ. Health, 80, 1-15 (2006).

34) Fukuchi Y., Miura Y., Nabeno Y., Kato Y., Osawa Y., Naito M., J. Atheroscler. Thromb., 15, 185-192 (2008).

35) Kageyama Y., Takahashi M., Nagafusa T., Torikai E., Nagano A., Rheumatol. Int., 28, 245-251 (2008).

36) Nishiyama I., Fukuda T., Oota T., J. Agric. Food Chem., 26, 64036407, (2005)

37) Yui S., Sasaki T., Miyazaki A., Horiuchi S., Yamazaki M., Arterioscler. Thromb., 13, 331-337 (1993).

38) Prior R. L., Gu L., Wu X., Jacob R. A., Sotoudeh G., Kader A. A., Cook R. A., J. Am. Coll. Nutr., 26, 170-181 (2007).

39) Fiorentino A., D’Abrosca B., Pacifico S., Mastellone C., Scognamiglio M., Monaco P., J. Agric. Food Chem., 57, 4148-4155 (2009).

40) Duttaroy A. K., Jørgensen A., Platelets, 15, 287-292 (2004)

41) Chang W. H., Liu J. F., Int. J. Food Sci. Nutr., 60, 709-716 (2009). 